\title{
GRUPO FAMILIAR NA CONVIVÊNCIA COM O DOENTE MENTAL: PROGRAMA DE EDUCAÇÃO EM SAÚDE ${ }^{1}$
}

\author{
FAMILY GROUP IN THE FAMILIARITY WITH THE MENTAL PATIENT: \\ HEALTHY EDUCATIONAL PROGRAM \\ GRUPO FAMILIAR EN LA CONVIVENCIA CON EL ENFERMO MENTAL
}

Sônia Maria Alves de Paiva*
Maguida Costa Stefanelli**
Evalda Cançado Arantes***

RESUMO: Em nossa experiência com doentes mentais, percebemos que tanto eles quanto suas famílias padecem do mal da desinformação. Esta pesquisa exploratória - descritiva, foi realizada com os objetivos de identificar as principais indagações dos familiares de pessoas com diagnóstico de esquizofrenia, com a finalidade de implementar e avaliar um plano de orientação às famílias de tais pacientes. Os dados do presente estudo foram obtidos em entrevistas com o familiar ou famílias e a análise dos mesmos permitiu a identificação das dúvidas e expectativas destes. Com base nestes resultados foi elaborado um plano de orientação aos familiares, que foi implementado em sessões grupais com as famílias, realizadas no ambulatório do hospital. A orientação realizada foi avaliada pela população do estudo como sendo importante para aquisição de conhecimento e melhor compreensão do comportamento do paciente, permitindo segurança nas relações com a pessoa doente e sobretudo, aprender a não excluí-lo do convívio familiar.

PALAVRAS CHAVE: E ducação em saúde; Saúde mental; Família.

\section{INTRODUÇÃO}

Há mais de dez anos a desinstitucionalização e reabilitação do doente mental passaram a fazer parte do "discurso" psiquiátrico e a receber atenção por parte das autoridades da área da saúde e de alguns profissionais nela envolvidos, em decorrência da chamada Reforma Psiquiátrica.

Apesar de todos os esforços desenvolvidos para a implementação da Reforma Psiquiátrica, as mudanças, com raras exceções, vêm sendo efetuadas de forma lenta com a reorganização e criação de diferentes modelos de atendimento na assistência psiquiátrica.

Esses modelos propostos, entretanto, não correspondem à demanda da população que deles necessita, dada as características biogeográficas, econômicas e culturais, entre outras, do nosso País. A falta de pessoal qualificado em número suficiente e a falta de vontade política que se expressa por meio da ausência de compromisso real com a criação de rede de atenção psicossocial, num desafio de inclusão dos mentalmente enfermos.

Quando nos deparamos com a família do doente mental, apesar dos esforços de profissionais da área específica, às vezes de forma organizada, com o apoio de órgãos públicos responsáveis pela saúde da população, apoio de organizações não-governamentais (ONGs) e mesmo de grupos isolados, o que sobressai é a falta de informação da mesma, além da luta para enfrentar o preconceito e a rejeição dos que a cercam, "o não saber o que fazer" e o "como agir" com a pessoa considerada doente mental.

\footnotetext{
Extraído e ampliado da dissertação de mestrado de Sônia Maria Alves de Paiva, intitulada "Orientação de Familiares de Pacientes com Diagnóstico de Esquizofrenia", submetida à Arguição na Escola de Enfermagem da Universidade de São Paulo, 1989. Apoio da FAPESP.

* Enfermeira. Mestre em Enfermagem. Enfermeira responsável pela Clínica de Repouso Mococa, Mococa / SP.

* Enfermeira. Orientadora. Professora Titular. Doutora em Enfermagem. Professora Visitante da Universidade Federal do Paraná. Membro do Grupo de Estudos Família, Saúde e Desenvolvimento (GEFASED).

*** Enfermeira. Doutora em Enfermagem. Professora do Departamento de Enfermagem Materno Infantil e Psiquiátrica da Escola de Enfermagem da Universidade de São Paulo.
} 
A doença mental ainda suscita mal-estar nas pessoas não só pelo medo do desconhecido, mas por tratar-se de algo carregado de crenças e preconceitos, principalmente quando falamos em esquizofrenia. Esta é uma condição multicausal que se manifesta por uma heterogeneidade de manifestações de comportamento que, na maioria das vezes, deixa a família perplexa, sem saber como agir e torna-se, também, um desafio para o profissional - não há receitas mágicas e tem-se de considerar a diversidade de manifestações que são peculiares a cada um.

Vários trabalhos, há algum tempo, vêm enfocando a importância do trabalho com a família quando há nesta um membro considerado doente mental. Os resultados da pesquisa de Fukuda (1978) mostraram que mesmo os familiares que haviam recebido alguma orientação consideraram-na insuficiente e, quando questionados, expressaram necessidade de receber informações sobre como lidar e como se relacionar com o paciente.

Carmo (1981) mostrou que a maioria dos familiares da população de seu estudo, não recebeu qualquer orientação durante a internação do paciente. Quando a receberam, essa foi realizada por médicos, assistentes sociais e atendentes de enfermagem, não correspondendo às expectativas daqueles familiares.

Battle et al. (1982) afirmaram que um programa de educação e orientação para o paciente, durante sua hospitalização, tem influência significativa na aceitação do plano de tratamento. Para tanto é preciso torná-lo efetivo. A readmissão de pacientes, freqüentemente desnecessária, é precipitada pela falta de orientação dos pacientes e de sua família para enfrentarem situações críticas.

Os estudos de Rose (1983) demonstraram que os familiares identificaram problemas relativos ao controle de comportamento do paciente e mostraram necessidades de informação quanto à doença mental, ao tratamento e ao futuro do paciente.

Esses resultados foram confirmados por Hughes et al. (1987) quando sugerem que os enfermeiros se reunam com a família do paciente imediatamente após sua admissão, para coletar informações acerca do mesmo, da dinâmica familiar e dos recursos oferecidos pela comunidade. Esses autores enfatizam que os enfermeiros devem assegurar orientações aos familiares, principalmente no planejamento do cuidado pós-alta. Afirmam que os pacientes, cujos familiares receberam alguma orientação, sofreram menor número de readmissões.

Concordando com Carneiro (1992), que considera a interação familiar como promotora da saúde mental dos membros da família, achamos de fundamental importância a atuação da enfermeira junto aos familiares dos pacientes com diagnóstico de esquizofrenia, não só para o bem-estar da família como o do próprio paciente. Afirma ainda que na equipe de saúde, a enfermeira é a que convive mais tempo com o paciente e é a que tem mais oportunidade de interagir com a família, seja em ambulatório ou em unidade de internação. É, portanto, a pessoa que pode orientar a família no momento em que esta vem à instituição de saúde para trazer o membro que julga estar doente, para consulta ou para visitá-lo quando internado em hospital.

Cordo (1996) ressalta a importância de se estimular o profissional de saúde a trabalhar em conjunto com a família. Koga (1997) enfatiza a importância de se oferecer à família orientação e assistência para que aceite a doença mental. Waidman; J ouclas; Stefanelli (1999) afirmam que deve-se propiciar à família do doente mental orientações e informações para que esta possa atuar como agente ressocializador e facilitador da integração da pessoa na família e na comunidade.

A idéia é reforçada por Sarraceno(1999), quando afirma que deve-se aumentar a estabilidade emocional no ambiente familiar e a capacidade dos familiares para interagirem com a pessoa doente, além de torná-los capazes de exprimirem os próprios sentimentos e necessidades de forma direta, facilitando a solução dos conflitos que possam surgir.

As afirmações destes autores corroboram o que percebemos em nossa experiência com doentes mentais, ou seja, sofrendo do mal da desinformação. Constatação esta que nos deu a coragem para ousar publicar 0 presente estudo, extraído da dissertação de Paiva(1989).

Esta decisão de publicá-lo agora foi fundamentada nas considerações acima, nas discussões entre as autoras e na validação dos resultados encontrados por Paiva (1989) junto a enfermeiras e docentes que estão atuando na área de enfermagem psiquiátrica, neste momento. Esta validação evidenciou a atualidade e a pertinência do estudo realizado e a necessidade da divulgação de experiências que envolvem a família como um todo ou familiares de doentes mentais, para que estes possam atuar como participantes no tratamento e recuperação do membro mais afetado e também na prevenção da doença, ou pelo menos minimizar seu efeito sobre os demais componentes da família. 
A validação foi efetuada, também, junto a familiares de pacientes esquizofrênicos internados em uma instituição psiquiátrica, por meio de levantamento, utilizando o mesmo instrumento de Paiva (1989). Verificamos novamente o quanto os resultados encontrados anteriormente pela pesquisadora, são atuais e vividos no dia-dia das famílias destes pacientes, apesar de todo o movimento da Reforma Psiquiátrica e os esforços para implementá-la.

\section{OBJ ETIVOS}

- Identificar a necessidade de orientação da família ou familiares de pessoas portadoras do diagnóstico de esquizofrenia, com base em suas expectativas.

- Implementar e avaliar um plano de orientação à família ou aos familiares de tais pessoas.

\section{MÉTODO}

Trata-se de uma pesquisa prática descritiva, realizada em duas etapas. Na primeira, foi realizado o levantamento das expectativas, dúvidas e dificuldades dos familiares em relação a um membro da familia portador do diagnóstico de esquizofrenia, para elaborar o plano de educação e orientação, a ser implementado na etapa seguinte. Já na segunda etapa, foi efetuada a implementação efetiva do plano de orientação aos familiares. Estamos utilizando família ou familiares, pois, muitas vezes as pessoas que participam do estudo, constituíam a família do paciente.

A pesquisa foi limitada a família ou familiares de pacientes esquizofrênicos, porque a esquizofrenia, além de ser o sofrimento psíquico mais comum, é caracterizada por um processo, extremamente complexo que provoca profunda modificação no modo de ser da pessoa, o que dificulta o seu cuidado e o convívio com ele.

\section{LOCAL}

0 estudo foi realizado em ambulatório de pacientes adultos de um hospital-escola psiquiátrico, estatal, do Município de São Paulo, que atende pessoas de ambos os sexos, em tratamento ambulatorial e em regime de internação.

\section{POPULAÇÃO}

Para a população deste estudo foram considerados os familiares ou a família de pacientes com diagnóstico de esquizofrenia que buscaram atendimento no ambulatório da instituição, campo de estudo, durante a fase de coleta de dados.

\section{CRITÉRIOS PARA SELEÇÃO DA POPULAÇÃO}

Fizeram parte do estudo os familiares ou a família de pacientes com diagnóstico firmado como esquizofrenia, pela equipe médica, que segue a Classificação Internacional das Doenças Mentais, com idade acima de 18 anos, com algum tempo de convivência com o mesmo, que o acompanhavam com freqüência às consultas médicas e que, estivessem aptos para fornecerem as informações necessárias sobre o paciente. Com base em estudo realizado pelo estatístico, foi possível definir que 42 familiares seria um número significativo para este estudo.

\section{ASPECTOS ÉTICOS}

Os respondentes foram orientados quanto a sigilo, anonimato, uso que se faria dos dados, participação voluntária com direito de interrompê-la se julgasse necessário; foi assumido também o compromisso de retorno dos dados para a população e a instituição. 


\section{INSTRUMENTOS DE COLETA DE DADOS}

No desenvolvimento do estudo foram utilizados três instrumentos: um formulário para entrevista com o familiar, incluindo questões referentes ao vínculo familiar, ao relacionamento dos familiares com o paciente e suas expectativas quanto ao tratamento, às informações que estes tinham a respeito da doença, do tratamento e que orientações gostariam de receber.

Após o resultado da primeira etapa, elaborou-se o conteúdo do plano de orientação em relação aos temas esquizofrenia, manifestações de comportamento apresentadas pelos pacientes diagnosticados como esquizofrênicos, cuidado e tratamento desses pacientes, segundo as expectativas e necessidades apontadas pelos participantes deste estudo.

Outro formulário foi utilizado para verificar a opinião dos familiares sobre o plano de orientação, averiguar se gostariam de obter mais alguma informação e, em caso positivo, se desejavam recebê-la em grupo ou individualmente.

A entrevista com familiares ou família foi realizada pela própria pesquisadora, em sala privada, e o formulário preenchido pela mesma, na presença do familiar. Ao final, era lido o registro das informações para assegurar a fidedignidade das mesmas.

A análise das respostas obtidas foi realizada com o agrupamento das mesmas segundo categorias emergentes das falas.

\section{RESULTADOS E COMENTÁRIOS}

Participaram deste estudo 42 pessoas do grupo familiar de pacientes esquizofrênicos, havendo predomínio de "mães". Os integrantes da população, em sua maioria $(91,2 \%)$, conviviam com o paciente há mais de 20 anos, sendo que a manifestação da doença, em sua maioria, ocorrera no período de um a quinze anos, o que nos permite supor que a população desse estudo estava habilitada a informar sobre o paciente, sua doença e tratamento, enfim, sobre o seu convívio com a doença mental.

\section{PRIMEIRA ETAPA}

As expectativas dos familiares ou da família, em relação ao tratamento foram agrupadas em cura $(42,9 \%)$; melhora $(40,8 \%)$; descrença no tratamento $(10,2 \%)$ e outras $(6,1 \%)$, como observado no gráfico 1 .

GRÁFICO 1 - Relação das respostas dos familiares quanto as expectativas dos familiares em relação ao tratamento.

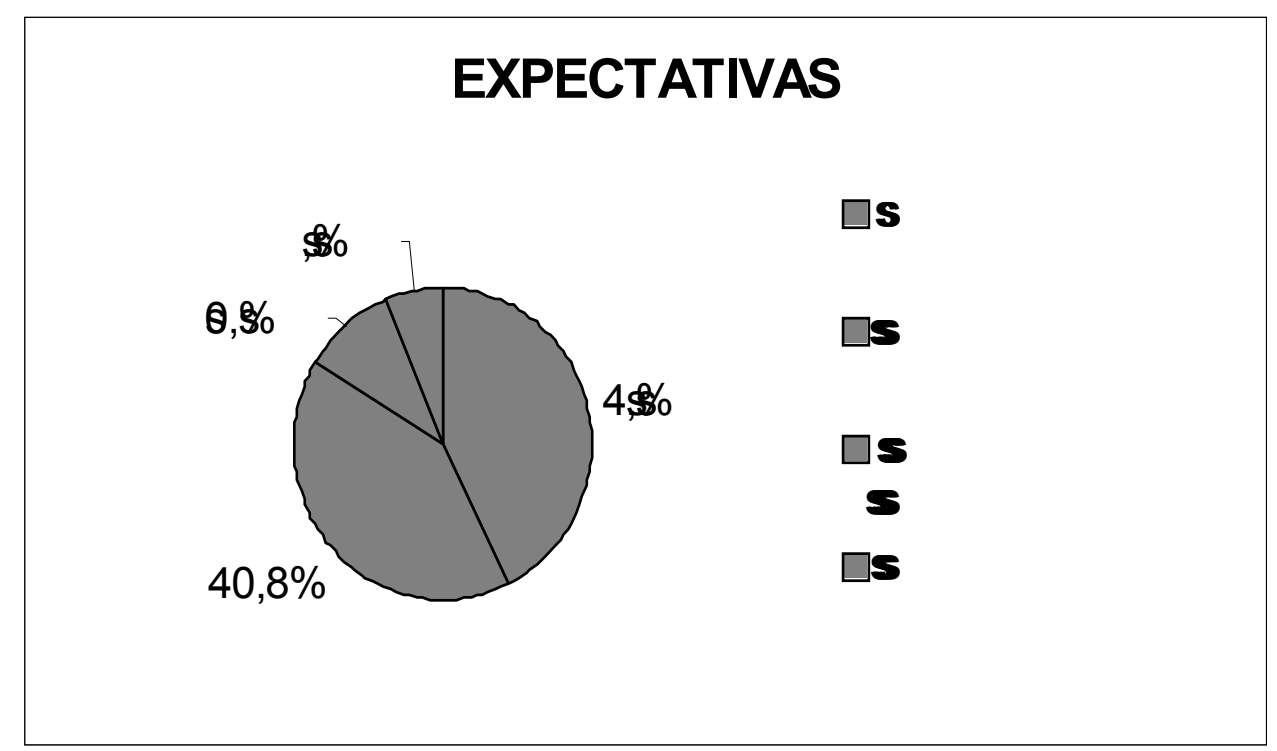


Em "cura" foram agrupadas algumas afirmações como: espera que o paciente sare; que se torne uma pessoa normal; que fique bom e não fique com seqüelas, entre outros.

Algumas respostas incluídas na categoria "melhora" foram: espera que o paciente melhore; que melhore porque a médica já disse; a doença não tem cura; que melhore e possa voltar a estudar.

Q uanto a "descrença no tratamento" referiram-se principalmente ao efeito da medicação, mencionando a demora do tratamento, quantidade excessiva de medicamento relacionado a não melhora do paciente e ainda apontaram a regressão no comportamento do paciente e que falta o médico descobrir o que ele tem.

Os familiares, em sua grande maioria $(95,9 \%)$, expressaram a necessidade de receber orientação e especificaram que gostariam de recebê-la sobre a doença, cuidado e tratamento, sendo as respostas agrupadas nas categorias: doença, cuidado e tratamento, apresentadas nos quadros a seguir.

QUADRO 1 - Relação das respostas dos familiares, de acordo com a orientação desejada, agrupadas na categoria DOENÇA.

Saber se a doença tem cura

Esclarecer qual é a doença do paciente

Saber porque o paciente ficou assim

Saber o que é esquizofrenia

Saber porque a doença deixa o paciente nervoso

Saber se o paciente vai poder ter vida normal, se casar, ter filhos

Saber se a doença é devida a falta de sexo

Esclarecer porque o "eletro" e Raio-X não acusam nenhuma alteração

Saber se a doença é dos "nervos"

Esclarecer se o problema do paciente é doença ou preguiça

Saber se vai ficar mais tempo internado

QUADRO 2 - Relação das respostas dos familiares de acordo com a orientação desejada, agrupadas na categoria: CUIDADO.

Como agir com o paciente

Saber se o paciente poderá ou não trabalhar

Como fazer para tirar o paciente da cama, fazê-lo sair de casa, sentir vontade de fazer

alguma coisa

Saber como agir com o paciente

Saber o que se pode conversar com o paciente

Obter informação sobre que atividade seria importante para o paciente

Saber se deve ter mais paciência com o paciente ou se deve tratá-lo com mais energia

Saber se está agindo correto com o paciente

Saber se deve dar responsabilidade para o paciente

Como perceber quando o paciente está ficando agressivo

Como fazer para tornar o paciente mais independente

QUADRO 3 - Relação das respostas dos familiares, de acordo com a orientação desejada, agrupadas na categoria: TRATAMENTO.

Saber até quando o paciente tomará a medicação

Saber para que serve a medicação

Informar-se sobre os efeitos colaterais da medicação

Saber qual o remédio é mais adequado para o paciente

Saber se uma conversa com o paciente, juntamente com a medicação, não o ajudaria

Saber se o paciente fará mais exames

Saber se a medicação provoca impotência

Saber se o paciente pode ingerir bebida alcoólica

Saber o que acontecerá, se o paciente parar de tomar a medicação

Saber se o paciente fará mais exame 
Q uanto às expectativas dos familiares sobre o tratamento predominou a expectativa de cura, conforme se verifica no gráfico 1.

Carmo (1981) relata que mais da metade da população do seu estudo expressou esperança de cura no tratamento. Esse é um aspecto importante, porque os familiares transmitem, de alguma forma, essa esperança para o paciente.

Com base na literatura e em nossas observações, acreditamos que essa expectativa dos familiares é percebida pelos pacientes, criando ansiedade nestes por não corresponderem às suas próprias expectativas.

Neste estudo, constatamos que os familiares esperam que o paciente volte a ser uma pessoa normal, possa trabalhar, se recuperar e não ficar com seqüelas.

Estes achados foram considerados importantes para reforçar a necessidade de se incluir num programa de orientação para familiares, informações quanto ao prognóstico da doença e quanto à limitação da capacidade do paciente, ressaltando que, com o tratamento de manutenção, o mesmo poderá desempenhar algumas tarefas.

Segundo Torrey (1983), o difícil para os familiares de pacientes esquizofrênicos é a aceitação da realidade da doença e a modificação de suas expectativas quanto a seu futuro e isto não significa que os familiares não devam ter nenhuma expectativa em relação ao doente, porém, reconhecerem que o paciente pode ficar com uma limitação de sua capacidade.

Mereness (1973) enfatizou a impotência do enfermeiro para ajudar os familiares a planejar o futuro do paciente de forma realista.

Nota-se, ainda no gráfico 1, que alguns familiares não acreditam na reabilitação do paciente.

Analisando as respostas acerca da orientação recebida sobre a doença, constatamos que apenas alguns deles haviam recebido algumas informações e estas, em sua maioria, haviam sido dadas por médicos e foram consideradas insuficientes. Em relação à orientação sobre a medicação, alguns familiares responderam que haviam recebido pouca informação.

Estes resultados chamaram a nossa atenção pela ausência de informações dadas pelos enfermeiros.

Carmo (1981) constatou em seu estudo que a maior percentagem de orientações foi dada por médicos e essas não correspondiam às expectativas dos familiares; este fato o levou a crer que o enfermeiro não ouve os pacientes e a família, talvez devido à insegurança por não saber resolver ou como ajudar na resolução dos problemas.

Pelos resultados deste estudo e da literatura enfocada, pôde-se concluir que os enfermeiros não estão dando o devido valor a esse aspecto assistencial, ou seja, o da educação em saúde.

Em relação às necessidades de receber orientação, a maioria demonstrou essa necessidade, embora alguns não soubessem dizer que informação gostariam de receber, sendo que poucos deles não revelaram interesse em recebê-la.

Outro fator importante a ser considerado é que, segundo Miles (1982), a doença mental afeta não apenas a qualidade de vida da família, mas também a saúde de seus membros; o clima de tensão emocional, a ansiedade e a responsabilidade pelo paciente têm efeitos sobre a saúde física e mental do grupo familiar.

Estes dados nos permitiram supor que a família não tem sido orientada de modo satisfatório e que esta é uma atividade do enfermeiro, que consta do Decreto n. 0 94.406, de 8 de junho de 1987 (BRASIL, 1987).

Com base nestes resultados, elaboramos um plano de educação em saúde para familiares de pacientes portadores de diagnóstico de esquizofrenia, segunda etapa deste estudo.

\section{SEGUNDA ETAPA}

\section{ELABORAÇÃO DO PLANO DE ORIENTAÇÃO}

Tendo como sustentação os grupamentos da primeira etapa e as respostas dadas pelos participantes do estudo foi elaborado o plano de orientação aos familiares ou a família. Como foram selecionados para este estudo, apenas familiares de pacientes com diagnóstico de esquizofrenia, o conteúdo do plano foi direcionado para atender as necessidades decorrentes de alterações mencionadas pela família, sendo seu conteúdo descrito sucintamente a seguir. 
I. Introdução.

1. O paciente como ser humano e suas necessidades humanas básicas.

2. Problemas vivenciados pelos familiares decorrentes da doença mental.

3. J ustificativa para a implementação do plano.

II. A doença e suas manifestações de comportamento.

1. Conceito de esquizofrenia, etiologia e evolução do quadro clínico.

2. Descrição das diferentes manifestações de comportamento que o doente pode apresentar, decorrentes do quadro clínico.

III. Cuidados a serem prestados pela família ao paciente que apresenta manifestações de comportamento decorrente de sintomas de esquizofrenia.

IV. Tratamento.

1. Importância da medicação.

2. Efeitos desejados.

3. Efeitos colaterais.

4. Cuidados a serem prestados a pessoa doente quando em tratamento por droga psicotrópica.

5. Orientação quanto a importância da participação da família no tratamento.

\section{IMPLEMENTAÇÃO DO PLANO DE ORIENTAÇÃO}

Após a elaboração do plano de orientação, optamos por fazer as orientações em grupo, porque é conhecida a tendência das famílias de doentes mentais se sentirem isoladas em sua experiência e de não terem com quem conversar sobre suas dúvidas e dificuldades pois, com base em nossa observação de que trabalhar com grupos é mais proveitoso porque propicia troca de experiência muito enriquecedora, entre os que estão em situação similar.

Cada reunião teve duração de uma hora e foram realizadas 13 reuniões. As orientações foram feitas por meio de exposição oral e discussão sobre o assunto com os familiares, que tiveram assim, oportunidade de participar da discussão a respeito dos temas abordados e de esclarecerem as dúvidas emergentes.

Para avaliar a opinião dos mesmos sobre a orientação recebida, foi realizada uma entrevista com cada familiar, utilizando um formulário específico para tratar destas questões. Com a finalidade de evitar a interferência das pesquisadoras nas respostas obtidas, essa entrevista foi efetuada por duas enfermeiras, devidamente orientadas.

\section{AVALIAÇÃO DO PLANO DE ORIENTAÇÃO}

$\mathrm{Na}$ análise da opinião dos familiares sobre o plano educativo, depreendemos que todos consideraram as informações importantes, no tocante à temática central, o que reforça a nossa crença de que um plano educativo e de orientação para familiares de pacientes, com diagnóstico de esquizofrenia, tem resultados expressivos porque os ajudam a aliviar suas tensões, a adquirirem alguns conhecimentos sobre a doença mental para melhor compreenderem o comportamento do paciente e porque proporcionam-lhes mais segurança no cuidado desse, ajudando-os a resolverem suas dificuldades no convívio com o paciente.

Ainda em relação a estes dados, alguns familiares disseram que iriam transmitir estas informações para outros membros da família que não aceitavam o comportamento do paciente; outros afirmaram que com a orientação recebida se sentiam mais seguros e aliviados e outro referiu que considerava a informação importante porque o ajudava a aceitar melhor o comportamento do paciente e a não exclui-lo do convívio familiar.

Pelas respostas obtidas quanto a forma de orientação que preferiam, em grupo ou individualmente, a maioria foi favorável à orientação coletiva. 
Percebeu-se que a maioria optou por orientação em grupo, o que chamou a nossa atenção para a importância dos grupos como fonte de apoio e orientação para os familiares de doentes mentais.

\section{CONSIDERAÇÕES FINAIS}

Percebemos que a família do doente mental tem dificuldades para lidar com ele, com a doença mental e com o tratamento por não terem informações suficientes para tal, portanto, suscitando atenção especial. No presente estudo, os resultados evidenciaram que as pesquisadoras foram bem aceitas pelos familiares e famílias, que demonstraram confiança nelas, quando da demonstração de conhecimento e do real interesse em ajudálos. Registramos, portanto, que a atuação do enfermeiro nos programas de educação em saúde para familiares é de fundamental importância.

Além disso, os resultados também evidenciaram que os familiares estavam carentes de informações e que as orientações que foram dadas por outros profissionais não os ajudaram suficientemente.

Estes achados foram considerados relevantes para chamar atenção dos enfermeiros que atuam na área de Saúde Mental, para que eles comecem a desenvolver conhecimentos e habilidades na implementação de programas de educação e orientação que incluam os familiares ou famílias, uma vez que as ações de enfermagem devem englobar a assistência ao doente, família e comunidade.

Temos de destacar que no conteúdo educativo foram abordados os temas mais comuns aos familiares, não se podendo ignorar, na continuidade de uma intervenção familiar, a necessidade de algumas orientações individuais, atendendo-se assim, as especificidades de cada um, tendo em vista a qualidade de vida e a visão do ser como um todo inserido em uma família singular.

Consideramos importante pois, que ao se implementar um plano de educação, este deve ser elaborado a partir do conhecimento das dúvidas e dificuldades sentidas pela família dos doentes e, se possível, com acompanhamento no domicílio.

É fundamental despertar a consciência dos trabalhadores de saúde mental para a importância do envolvimento da família de pessoas com diagnóstico de esquizofrenia nos projetos de tratamento e reabilitação, tornando-a co-responsável nesse processo de busca de uma convivência saudável.

Cabe à nós enfermeiros acreditarmos na abordagem biopsicossocial, destinada ao tratamento e à prevenção da doença mental, capaz de melhorar as condições de vida da pessoa doente, propiciando o respeito a sua dignidade de cidadão, num processo de humanização e transformação, que beneficie não só o paciente mas a família, assim como todos os sujeitos sociais envolvidos nesse processo.

ABSTRACT: In our experience with mental patients, we noticed that both then and their families suffer for lack of information. This research was realized with the objectives of identify the main inquiries of the relatives of the people with schizofrenic diagnosis and based on its expectations implement and assess a plan of orientation to the families of such patients. The data of this study were obtained in interview with the relative or families and their analisis permited the identification of the their doubts and expectation. Based on these results a plan of orientation to the relatives, which was implemented in group with the families, realized in the ambulatory of the hospital. The orientation was evaluated by the population of this study as important to the acquisition of knowledge and better comprehension of the behavior of the patient, permiting security in the relation with the sick person and above all, to learn not to exclude him from the family.

KEY WORDS: Healthy education, Mental health, Family.

RESUMEN: En nuestra experiencia como profesores de salud mental, percibimos que tanto ellos como sus familias tienen el mal de la desinformación. Esta investigación fué realizada con el objetivo de identificar las principales preguntas de los familiares de personas con diagnóstico de esquizofrenia y, con base en sus expectativas, implementar y evaluar un plan de orientación a sus familias. Los datos del presente estudio fueron obtenidos através de entrevistas con el familiar o familiares y su análisis permitió la identificación de las inquietudes. Con base en los resultados se elaboró un plan de orientación a los familiares, el cual se implementó 
mediante trabajos de grupo con las familias, los cuales se realizaron en la consulta externa del hospital. La orientación recibida fué evaluada por la población del estudio como importante para adquirir conocimiento y comprender mejor el comportamiento del paciente, permitiendo seguridad en las relaciones con la persona enferma y antetodo, aprender a no rechazarlo en la convivencia familiar.

PALABRAS CLAVE: Educación en salud, Salud mental, Familia.

\section{REFERÊNCIAS BIBLIOGRÁFICAS}

1. BATTLE, E. H. et al. Self - medication among psychiatric patients and adherence after discharge. J . Psychosoc. Nurs. Ment. Health Serv., Thorofare, v. 20, n. 5, p. 21-28, may 1982.

2. BRASIL, LEIS, DECRETOS, etc. Decreto no 94.406 de 8 de junho de 1987. Diário Oficial, Brasília, 9 de junho de 1987. Seção I, p. 8.853-55. Regulamenta a lei oo 7.498 de 25 de junho de 1986, que dispõe sobre o exercício de enfermagem e da outras providências.

3. CARMO, D. R. Contribuição ao estudo da integração da família de pacientes egressos de hospitais psiquiátricos. Ribeirão P reto, 1981. 88p. Dissertação (Mestrado). Escola de Enfermagem de R ibeirão Preto, Universidade de São Paulo.

4. CARneiRO, T. F. Família e saúde mental. Psicol. Teor. Pesq. v. 8, supl., p. 485-493, 1992.

5. CORDO, M. A. A família do doente mental: Hospitalidade, Lisboa. n. 236/37, p. 107-12, 1996.

6. FUKUDA, I. M. K. Dificuldades relacionadas com o retorno a comunidade de 39 pacientes esquizofrênicos após uma primeira internação em hospital psiquiátrico. São Paulo, 1978. Dissertação (Mestrado) - Escola de Enfermagem, Universidade de São Paulo.

7. HUGHES, L. et al. Does the family make a difference. J . Psychosoc. Nurs. Ment. Health Serv., Thorofare. v. 25, n. 8, p. 9-13, Aug. 1987.

8. KOGA, M. Convivência com a pessoa esquizofrênica: sobrecarga familiar. Ribeirão Preto, 1997. Dissertação (Mestrado) -Escola de Enfermagem de Ribeirão P reto, Universidade de São Paulo.

9. MERENESS, D., Elementos de enfermería psiquiatrica. México : Prensa Médica, 1973.

10. MILES, A. O doente mental na sociedade contemporânea. Rio de J aneiro : Zahar, 1982. Cap. 5, p. 111-134.

11. PAIVA, S. A., Orientações de familiares de pacientes com o diagnóstico de esquizofrenia. São Paulo, 1989. Dissertação (Mestrado) - Escola de Enfermagem, Universidade de São Paulo.

12. ROSE, L. E. Undestanding mental illness; the experience of families of psychiatric patients. J . Adv. Nurs., Oxford. v.8, n. 6, p. 507-511, Nov. 1983.

13. SARRACENO,B., Libertando identidade: da reabilitação psicosocial à cidadania possível. Belo Horizonte : Tecorá, 1999.

14. TORREY, E. F. Surving schizophrenic: a family manual. New York : Harper \& Row, 1983.

15. WAIDMAN,M. A. P. et al. Família e doença mental. Fam. Saúde Desenv., Curitiba, v.1, n. 1/2, p. 27-32, jan/dez. 1999. 